\title{
Fremdsprachliche Textkompetenz entwickeln
}

von Dagmar Knorr und Antonella Nardi (Hg.), Frankfurt am Main: Peter Lang, 2011, ISBN 978-3-631-60967-5, 301 S., €49,80

Rezensiert von Manuela Caterina Moroni, Trento

Im Mittelpunkt des vorliegenden Sammelbands stehen die Vermittlung und Evaluation von Textkompetenz in Deutsch als Fremdsprache. Dieses Thema wird in vier Kapiteln erschlossen: 1. Theorie der Textkompetenz, 2. Textkompetenz in der Domäne Wissenschaft, 3. Didaktik der Textkompetenz und 4. das Erwerben von Textkompetenz.

Die Beiträge von Ehlich, Portmann-Tselikas und Hornung eröffnen den Band mit der theoretischen Kontextualisierung (Teil 1). In seinem thesenartig formulierten Aufsatz erläutert Ehlich, in welchem Verhältnis Sprache, Text und Kultur zueinander stehen. Dabei bilden die von ihm formulierten Thesen „Schritte“ in einem Gedankengang, der dem Leser u.a. deutlich macht, dass Texte und Textarten komplexe Produkte einzelner Kulturen sind. Daraus folgt, dass die Schrift- bzw. Textkompetenz eine zentrale Kulturtechnik bildet und dass Schrifterwerb eindeutig nicht auf den Erwerb des lateinbasierten Systems und der Grundzüge seiner Kombinatorik reduziert werden kann (S. 17). Sprachen lassen sich nach Ehlich in Kulturbünde gruppieren. Die Zugehörigkeit zweier Sprachgemeinschaften zum selben Kulturbund ist für Gemeinsamkeiten bei der Textualität der beiden Sprachen verantwortlich. Andererseits sorgt dies auch dafür, dass wichtige Unterschiede zwischen den Kultursprachen desselben Bundes schwieriger zu ermitteln sind bzw. sich hinter scheinbaren Ähnlichkeiten verbergen. In diesem Zusammenhang stellt nach Ehlich ,eine die Wörter und ihre „falschen Freunde“ wie die Elementarsyntax übersteigende Analyse, Vergleichung und hermeneutisch fundierte Charakterisierung“ von Texten und Textarten von Sprachen desselben und unterschiedlicher Kulturbünde ein wichtiges Desiderat der Forschung dar (S. 22).

Portmann-Tselikas legt dann in seinem Aufsatz die Grundbausteine für eine Theorie der „Wissenschaftlichkeit“ von Texten, indem er der Frage nachgeht, welche Elemente eines Textes für seine „Wissenschaftlichkeit“ konstitutiv sind. Dabei schlägt er anhand einer exemplarischen Analyse eines wissenschaftlichen Aufsatzes so genannte „mesostrukturelle Einheiten“ als Grundeinheiten bzw. „unmittelbare Konstituenten“ 
von wissenschaftlichen Texten vor (S. 31). Diese betrachtet er als minimale kommunikative Muster, die imstande sind, eine komplexe Mitteilung zu machen ( $\mathrm{S}$. 29), was wiederum definiert wird als versprachlichte Handlungsschritte, durch die ein Autor wissenschaftlich handelt. Zum Beispiel kann eine für die Wissenschaft prototypische Handlungsstruktur nach Portmann-Tselikas aus drei mesostrukturellen Elementen bestehen: (i) der Problematisierung einer fremden Position, (ii) der eigenen Re-Positionierung und (iii) der Präsentation von Forschungsergebnissen. Erst im Hinblick auf die mesostrukturelle Ebene sollen dann die mikrostrukturellen sprachlichen Elemente ermittelt werden, die ein Autor beim Formulieren einsetzt, d.h. die Mikrostruktur (zum Beispiel einzelne für die Wissenschaftssprache typische Formulierungen) ist erst als Teil einer Mesostruktur zu analysieren, durch die Autoren wissenschaftliche Arbeit leisten und eigene originelle Erkenntnisse erarbeiten (S. 40).

Hornung bringt mit ihrem Aufsatz eine Dimension des Fremdsprachenlernens in die wissenschaftliche Diskussion zur fremdsprachlichen Textkompetenz ein, die auf den ersten Blick nicht dazugehört: die prosodische Dimension, die „Lautwelt" der Fremdsprache. Dabei hat Prosodie als allererster Zugang zur Fremdsprache doch viel mit dem Thema des Sammelbandes zu tun. Hornung argumentiert überzeugend für eine Fremdsprachendidaktik, die analog zum Mutterspracherwerb das „Sich-Hineinhören“ als prioritär betrachtet. Dies gilt als Voraussetzung für „einerseits eine Sprachproduktion und für andererseits ein Lesen, das sich von der Fixierung auf wörtliches Übersetzen und muttersprachliches Intonieren frei machen kann“" (S. 64). Hierzu berichtet Hornung von einer Unterrichtsmethode, die an der Universität Modena mit Nullanfängern praktiziert wird, bei dem mit authentischen gesprochensprachlichen Daten versucht wird, das Ohr der Deutschlerner von Anfang an zu schulen.

Insgesamt erscheint der theoretische Teil gelungen. Er bietet eine gute Grundlage für die in den nächsten Teilen folgende Diskussion. So wird in Ehlichs Aufsatz die Relevanz des Themas des Bandes auf prägnante Weise begründet. Der Begriff der Mesoebene von Portmann-Tselikas wird in mehreren Aufsätzen des Bandes wiederaufgenommen. Dort wird deutlich, dass dessen Operationalisierung bei der Analyse von wisseschaftlichen Texten nicht immer unproblematisch ist. Hier ist offensichtlich weitere, vor allem empirische Forschung notwendig. Hornungs Beitrag zur Rolle der Prosodie für die DaF-Didaktik und die Heranführung von DaF-Lernern an mündliche und schriftliche Texte scheint wie oben schon erwähnt nur auf den ersten 
Blick einen marginalen Aspekt anzusprechen. Dies liegt daran, dass Prosodie bisher wenig beachtet wurde, was auch daran zu erkennen ist, dass im dem Band selbst an Hornungs Überlegungen kaum angeknüpft wird.

Die Aufsätze von Roncoroni, Stezano Cotelo, Carobbio und Heller behandeln dann die Textkompetenz in der Domäne der Wissenschaftssprache (Teil 2). Bei Roncoroni und Stezano Cotelo steht der didaktische Aspekt im Vordergrund. Roncoroni ermittelt anhand eines Korpus von Hausarbeiten italophoner Studierender deren Schwierigkeiten bei der Verwendung von Mitteln der Textstrukturierung und der Leserorientierung. Die im Korpus ermittelten Schwierigkeiten lassen auf kultur- und sprachspezifische Präferenzen der italienischen Studierenden schließen, die auf Unterschiede in der kognitiven Verarbeitung hindeuten könnten. Die von Roncoroni festgestellten Abweichungen zwischen den Hausarbeiten italophoner Studierender und den deutschen Expertentexten weisen auf zentrale Aspekte hin, bei denen in einer Didaktik des Deutschen als fremder Wissenschaftssprache an italienischen Universitäten angesetzt werden sollte. Stezano Cotelo setzt sich zum Ziel, das Verfassen wissenschaftlicher Texte „in überschaubare Aufgaben zu zerlegen“ (S. 92), und zu ermitteln, wie diese Aufgaben von ausländischen Studierenden in ihren Hausarbeiten bewältigt werden. Aus der Analyse leitet die Autorin innovative Strategien zur Vermittlung von Textkompetenz im Bereich Deutsch als Wissenschaftssprache insbesondere für die Verfassung von Hausarbeiten ab.

Carobbio und Heller konzentrieren sich auf die Ermittlung von Eigenschaften unterschiedlicher wissenschaftlicher Textsorten. Carobbio untersucht aus funktionalpragmatischer Sicht die konstitutiven Handlungsphasen von Einleitungen und Schlüssen in der Diskursart wissenschaftlichen Vortragens, indem sie prüft, inwiefern diese mit den in der Literatur besser erforschten Handlungsphasen von Einleitungen und Schlüssen in wissenschaftlichen Aufsätzen übereinstimmen. Carobbios Analyse von einem Korpus von Vorträgen zu soziologischen Themen ergibt, dass sich Einleitungen und Schlüsse im wissenschaftlichen Vortrag durch spezifische Handlungskonstellationen auszeichnen, die durch den mündlich-situativen Charakter des Vortrags bedingt sind. Die für die Textart wissenschaftlicher Aufsatz erarbeiteten Handlungsphasen lassen sich also zum großen Teil nicht auf den wissenschaftlichen Vortrag übertragen. Heller ermittelt anhand einer wissenschaftlichen und einer an das Publikum des Feuilletons der FAZ gerichteten Rezension einer 
literaturwissenschaftlichen Monographie makro-, meso- und mikrostrukturelle Eigenschaften der beiden Texte. Typische Verfahren der Popularisierung wissenschaftlicher Inhalte werden hier aufgezeigt: Die Rezension der FAZ zeichnet sich durch eine ausgeprägte Reduktion der Informationsfülle bzw. -dichte, eine Vereinfachung wissenschaftlicher Inhalte und eine starke Tendenz zur Personalisierung aus. Mit Rückgriff auf Portmann-Tselikas' Theorie der Mesostruktur zeigt Heller, dass die für die Wissenschaft typischen mesostrukturellen Elemente in der wissenschaftlichen Rezension aber nicht in dem FAZ-Text nachweisbar sind. Die Dichotomie „Wissenschaftlichkeit vs. Popularisierung“ lässt sich auch auf der Mikrobene bei der Verbsemantik feststellen.

Die Beiträge dieses zweiten Teils enthalten Herangehensweisen an wissenschaftliche Texte bzw. Diskurse, die für die Didaktik des Deutschen als fremden Wissenschaftssprache in der Auslandsgermanistik als wegweisend dienen sollten. Jeder der Beiträge bietet genug Material und Anregungen zur Gestaltung universitärer Veranstaltungen für die Germanistik und DaF auf unterschiedlichem Niveau an.

Die Didaktik der Textkompetenz (Teil 3) wird in den Beiträgen von Knorr, Nardi und von Gunten erarbeitet. In den Aufsätzen von Knorr und Nardi steht die Textkompetenz im Bereich der Lehrerausbildung an italienischen Universitäten im Vordergrund. Knorr behandelt die bisher wenig erforschte Textart des Randkommentars. Mit Randkommentaren müssen zukünftige DaF-Lehrer die Texte ihrer Schüler korrigieren bzw. Textartenwissen und metasprachliche Inhalte vermitteln. Diese Kompetenz muss daher auch Gegenstand der Lehrerausbildung sein. Hierfür erarbeitet Knorr eine didaktische Methode zur gegenseitigen Produktion und Rezeption von Randkommentaren. Lehramtstudierende schreiben in Teams Randkommentare zu Texten von Kollegen und bearbeiten den eigenen Text nach den Randkommentaren anderer Kollegen. Dies erlaubt, dass „Gedanken expliziert und Ideen ausgehandelt werden, die beim Ein-Personen-Schreiben im Kopf bleiben“" (S. 171). Knorr zeigt an authentischen Daten, welche Art von Randkommentaren produziert werden und ob und wie diese aufgenommen werden. Hieraus leitet sie eine vielversprechende Didaktik der Textkommentierungskompetenz ab. Nardis Aufsatz stellt den (gelungenen) Versuch dar, einer der am Anfang des Sammelbands formulierten Thesen von Ehlich gerecht zu werden und zwar der, dass Textkompetenz nicht bloß mit der Beherrschung ortographischer und grammatischer Regeln gleichgesetzt werden kann. Am Beispiel 
einer Lernbiographie des Deutschen einer italienischen Lehramtstudierenden entwickelt sie einen neuen Ansatz zur Evaluierung von Textproduktionen im Rahmen der Deutschlehrerausbildung. Dabei kombiniert sie die Gütekriterien des Zürcher Forschungsprojekts „Sprachfähigkeiten“ aus den 90er Jahren und den funktionalpragmatischen Ansatz. Die Kombination dieser zwei Ansätze erlaubt es, die Textkompetenz auf Makro-, Meso- und Mikroebene im Hinblick auf die pragmatischen Qualitäten des Textes $\mathrm{zu}$ beschreiben und bewerten. So wird mit Texten nicht fehlerorientiert umgegangen, sondern diese werden etwa auf ihre Angemessenheit im Hinblick auf die kommunikative Situation, die Absicht des Autors und die Adressatenführung analysiert.

Auch von Gunten beschäftigt sich mit funktional-pragmatisch orientierten Bewertungskriterien. Ihr Hauptziel ist, Textproduktionen von Zweitklässlern der deutschen Schweiz auf ihre pragmatische Qualität hin zu beurteilen. Hierfür entwickelt sie einen Kriterienkatalog anhand eines Korpus von schriftlichen Handlungsanleitungen. Dabei berücksichtigt sie die explizite Nennung der Textfunktion, die Überschrift, Anfang- und Schlussmarkierung, die Vollständigkeit des Informationsgehaltes, das instruktionsspezifische Sprachhandeln und den Realitätsbezug hinsichtlich der Adressaten, um nur einige Kriterien zu nennen (S. 202 ff.). An von Guntens Analyse wird deutlich, dass die Handlungsanleitung, die sonst in der Schule vor allem zugunsten der Textart Erzählung wenig Beachtung findet, sehr gut geeignet ist, um Schulkindern die Handlungsbezogenheit des Schreibens zu vermitteln. Die Korpusdaten lassen ferner auch Aufschlüsse hinsichtlich möglicher Unterschiede bzw. Analogien bei der Textkompetenz zwischen ein- und mehrsprachigen Kindern ziehen.

Die Beiträge von Knorr, Nardi und von Gunten tragen wirkungsvoll dazu bei, den Aspekt der Bewertung bzw. Beurteilung von Textproduktionen in den Vordergrund zu rücken, was in der germanistischen Didaktikforschung meist eher vernachlässigt wird. An dieser Stelle sei eine Anmerkung zum Aufbau bzw. Zusammenstellung des Sammelbandes erlaubt: Die Tatsache, dass der Aufsatz von von Guntens den Spracherwerb bei Schulkindern zum Gegenstand hat, könnte seine Rezeption beeinträchtigen, da der gesamte Band ansonsten die Textkompetenz im universitären Bereich als Schwerpunkt hat. 
Lombardi, Jammernegg Flinz und Jentges beschäftigen sich mit der Vermittlung fachsprachlicher Textkompetenzen im DaF-Bereich (Teil 4). Diese stehen im Mittelpunkt zahlreicher BA- und MA-Studiengänge, die im Zuge der Bologna-Reform an italienischen Universitäten eingerichtet wurden. Lombardi erarbeitet ein innovatives experimentelles Unterrichtsmodul, das in erster Linie auf den Erwerb von methodischem Wissen abzielt. MA-Studierende werden zur selbstständigen Arbeit mit Fachtextkorpora mit der Software WordSmith Tools angeleitet. Bei der Projektarbeit besonders thematisiert wird vor allem, wie die Quellen der Fachtexte ausgewählt und wie und zu welchem Zweck die Fachtextkorpora aufbereitet werden sollen (S. 221). Die Arbeit mit Korpora erlaubt ferner, durch ein forschendes Lernen ,textsortenspezifische Wortbildungsmuster systematisch zu erfassen und fachgebietsmarkierte Bedeutungen zu rekonstruieren“ (S. 232), die für den Beruf unabdingbar und dennoch keinem vorgefertigten Lehrbuch $\mathrm{zu}$ entnehmen sind. Bei Jammernegg geht es um die Erforschung und Vermittlung von relevanten Textmustern im Rahmen des MAStudiengangs für Öffentlichkeitsarbeit. An einem Korpus von deutschen und italienischen Texten aus dem Bereich der Öffentlichkeitsarbeit ermittelt die Autorin deren relevante Textmuster. Dabei macht sie interessante kulturell bedingte Unterschiede sichtbar, die sich auf der Marko-, Meso- und Mikrostruktur der Texte niederschlagen. So zeigt Jammernegg zum Beispiel, dass sich auf der Mesoebene italienische PR-Texte durch eine Reihung von Nebenthemen kennzeichnen, die durch Kohäsionsmittel (z.B. Konnektoren) miteinander und mit dem Hauptthema verbunden werden, wobei aber oft die Kohärenz vernachlässigt wird (S. 254). Bei der thematischen Progression in den deutschen Texten steht demgegenüber die Kohärenz im Vordergrund. Flinz entwickelt für die DaF-Didaktik im BA-Studiengang „Tourismuswissenschaft“ ein Modell zur Analyse der Textsorte Unterkunftsbeschreibung. Das Modell richtet sich an DaF-Anfänger und zielt darauf ab, die Studierenden durch ein inferenzgeleitetes Textverstehen an den Text bzw. an die deutsche (Fach)Sprache heranzuführen. Durch die Analyse der Makrostruktur eines Korpus von Unterkunftsbeschreibungen zeigt Flinz überzeugend, dass sich fachsprachliche Elemente bereits bei Anfängern vermitteln lassen und dass das inferentielle Textverstehen anhand einer aus der L1 bekannten hochstandardisierten Textart didaktisch sehr sinnvoll ist. 
Auf einen informationsstrukturellen Aspekt der Textkompetenz geht schließlich der Aufsatz von Jentges ein. Anhand der Analyse eines Korpus von Texten niederländischer DaF-Lerner zeigt Jentges, dass eine „unpassende“ Besetzung der satzinitialen Position in der Mehrheit der Fälle für einen zusammenhanglosen und argumentationslosen Eindruck verantwortlich ist (S. 239). In den Lerner-Texten aus Jentges’ Korpus wird die satzinitiale Position viel zu oft mit Subjekten besetzt (245). Dies ist möglicherweise auf die Übertragung eines L1-Musters auf die L2 zurückzuführen. So bietet sich nach Jetges an, die Besetzung dieser Position und den Themenkomplex der Informationsstruktur, auch in kontrastiver Perspektive, im DaF-Unterricht zu thematisieren. Die Aufsätze aus diesem letzten Teil runden das Buch insofern ab, als dort konkrete Vorschläge formuliert werden, wie man mit hohen wissenschaftlichen Standards dem Gebot der Berufs- bzw. Praxisbezogenheit der nicht immer begrüßten Bologna-Erklärung gerecht werden kann.

Insgesamt haben Knorr und Nardi hier einen Sammelband herausgegeben, in dem innovativ und praxisbezogen an das Thema Textkompetenz herangegangen wird und der neue, didaktisch nutzbare Erkenntnisse zu zahlreichen Textsorten enthält. 